\title{
Normas básicas de seguridad durante el manejo de equipos de radiaciones no ionizantes
}

\section{Safety basic rules when handling non-ionizing radiation equipment}

\author{
Rosa María Armida Bretones, Pilar Barbero del Palacio, Paz Uribe Llopis, M. ${ }^{a}$ Luisa Valle Roble, M. ${ }^{a}$ Dolores \\ Mínguez Martínez, Covadonga Caso Pita
}

\section{Unidad de Gestión Clínica del Servicio de Prevención de Riesgos Laborales - Área Centro - Hospital Clínico "San Carlos".} Madrid. España.

Recibido: 07-02-12

Aceptado: 14-02-12

\section{Correspondencia}

Rosa María Armida Bretones

Servicio de Prevención de Riesgos Laborales

Hospital Clínico "San Carlos"

Martín Lagos, s/n

28040 Madrid. España.

Tfno: 696206949

E-mail: rarmida.hcsc@salud.madrid.org

Resumen

La preocupación creciente de los trabajadores hacia los posibles efectos nocivos de la exposición a campos electromagnéticos, está suponiendo una percepción desproporcionada de los riesgos derivados de la exposición.

En la actualidad, no existe evidencia científica sobre dichos efectos nocivos, siendo aconsejable la vigilancia sobre las exposiciones que están fuera de los límites de seguridad.

Desde este Servicio de Prevención y en colaboración con el Servicio de Física Médica, hemos elaborado un procedimiento que garantice, mediante criterios preventivos básicos, la seguridad y salud de los trabajadores que manejan equipos emisores de radiaciones no ionizantes, en nuestro hospital y centros de especialidades.

Para la elaboración de este procedimiento hemos revisado la literatura científica relativa a los efectos de los campos electromagnéticos sobre la salud, reuniones periódicas de trabajo entre ambos servicios, identificación y ubicación de los equipos de radiaciones no ionizantes, y asesoramiento por expertos en la materia.

El procedimiento establece medidas de control para los trabajadores, y medidas de control para los equipos.

Con la finalidad de conseguir una buena difusión e implantación de dicho procedimiento, hemos elaborado dípticos y carteles, donde reflejamos las medidas de control para trabajadores y equipos: Onda Corta, Microondas, Magnetoterapia, Resonancia Magnética Nuclear, Láser y Ultravioleta

El procedimiento con sus carteles explicativos ha sido distribuido específicamente por servicios, realizando formación e información de los trabajadores que maneja dichos equipos

Med Segur Trab (Internet) 2012; 58 (226) 67-78

Palabras clave: Radiaciones No Ionizantes. Riesgo. Exposición laboral. Procedimiento. Normas de Seguridad. 
Abstract

Employee increasing concern about possible dangerous effects of the exposure to electromagnetic fields is meaning a distorted perception of the exposure related risks.

Currently, there is no scientific evidence of the dangerous effects mentioned although surveillance over the exposures out of the safety limits is recommended.

Within this Prevention Department in cooperation with The Medical Physics Department a procedure based on basic preventive criteria has been elaborated to guarantee health and safety of the employees who handle non-ionizing radiation emitting equipment in our hospital and specialized centers.

To draw the procedure: scientific literature related to the electromagnetic fields effects over health has been checked, periodical working meetings have been held between both above mentioned departments; non-ionizing radiation equipment have been identified as well as the places they are based or used; and expert people advice has been used.

The procedure sets control and follow-up measurements both for people and equipment such as follows: Shortwave, microwave and magnetic therapy, Nuclear magnetic resonance, Laser, Ultraviolet radiation.

The procedure and illustrative posters have been deployed to the linked departments, the information and training having been given to the employees who work with kind of equipment

Med Segur Trab (Internet) 2012; 58 (226) 67-78

Key words: Non-ionizing radiation. Risk. Labor exposure. Procedure. Safety rules. 


\section{INTRODUCCIÓN}

El creciente incremento en la preocupación de los trabajadores, hacia cuestiones relacionadas con eventuales efectos nocivos derivados de la exposición a campos electromagnéticos, ha dado lugar a una percepción desmesurada de los posibles riesgos a dichas exposiciones.

Si bien, es cierto que la actual base experimental y epidemiológica sobre los posibles efectos nocivos todavía es limitada, algunos piensan que existen en ella indicios, que aconsejarían prestar atención a posibles efectos de exposiciones crónicas a intensidades próximas o inferiores a los límites de seguridad.

En fase de investigación, entre tanto y a la espera de resultados concluyentes, este Servicio de Prevención adquiere el compromiso, de poner los medios para facilitar el cumplimiento de las recomendaciones de ICNIRP 1998 (Comisión Internacional de Protección en Radiaciones no Ionizantes) y de informar a los trabajadores de cómo y porqué deben cumplir las citadas recomendaciones, así como la realización de reconocimientos de salud periódicos.

\section{OBJETIVOS}

El objetivo principal es elaborar, junto con el Servicio de Física Médica, un Procedimiento que garantice, mediante criterios preventivos básicos, la seguridad y salud de los trabajadores/as que manejan equipos emisores de radiaciones no ionizantes, en el Hospital Clínico y los centros Pabellón 8, Modesto Lafuente y Avenida de Portugal.

\section{MATERIAL Y MÉTODOS}

- Revisión de evidencia científica acerca de los potenciales efectos de los CEM (campos electromagnéticos) sobre la salud.

- Reuniones de trabajo periódicas, con el Servicio de Física Médica del hospital para la elaboración del contenido técnico del Procedimiento.

- Reuniones del grupo de trabajo del Servicio de Prevención, estableciendo los criterios preventivos básicos para la elaboración de los documentos.

- Toma de datos: identificación actualizada de todos los equipos de radiaciones no ionizantes y su lugar de ubicación.

- Asesoramiento para la elaboración del Procedimiento de expertos en la materia (Sociedad Española de Protección Radiológica).

\section{RESULTADOS}

Elaboración del Procedimiento que concluye estructurado de la siguiente forma:

- Medidas de control para los trabajadores:

- Realización de reconocimientos médicos periódicos y específicos, al personal expuesto.

- Formación/información a los trabajadores.

- Asesoramiento y dotación de EPI adecuados.

- Medidas de control para los equipos:

- Correcta señalización de precaución.

- Mantenimiento preventivo para los equipos.

- Mediciones ambientales de radiación no ionizante anuales. 
- Elaboración de cuatro dípticos y seis carteles informativos, por parte del Servicio de Prevención, que darán difusión de las medidas de control para los trabajadores y para los siguientes equipos:

- Onda Corta, Microondas y Magnetoterapia

- Resonancia Magnética

- Láser

- Ultravioleta

Estos dípticos y carteles informativos fueron sometidos a evaluación por parte del grupo de trabajo de la Sociedad Española de Protección Radiológica (SEPR), quien avaló su contenido.

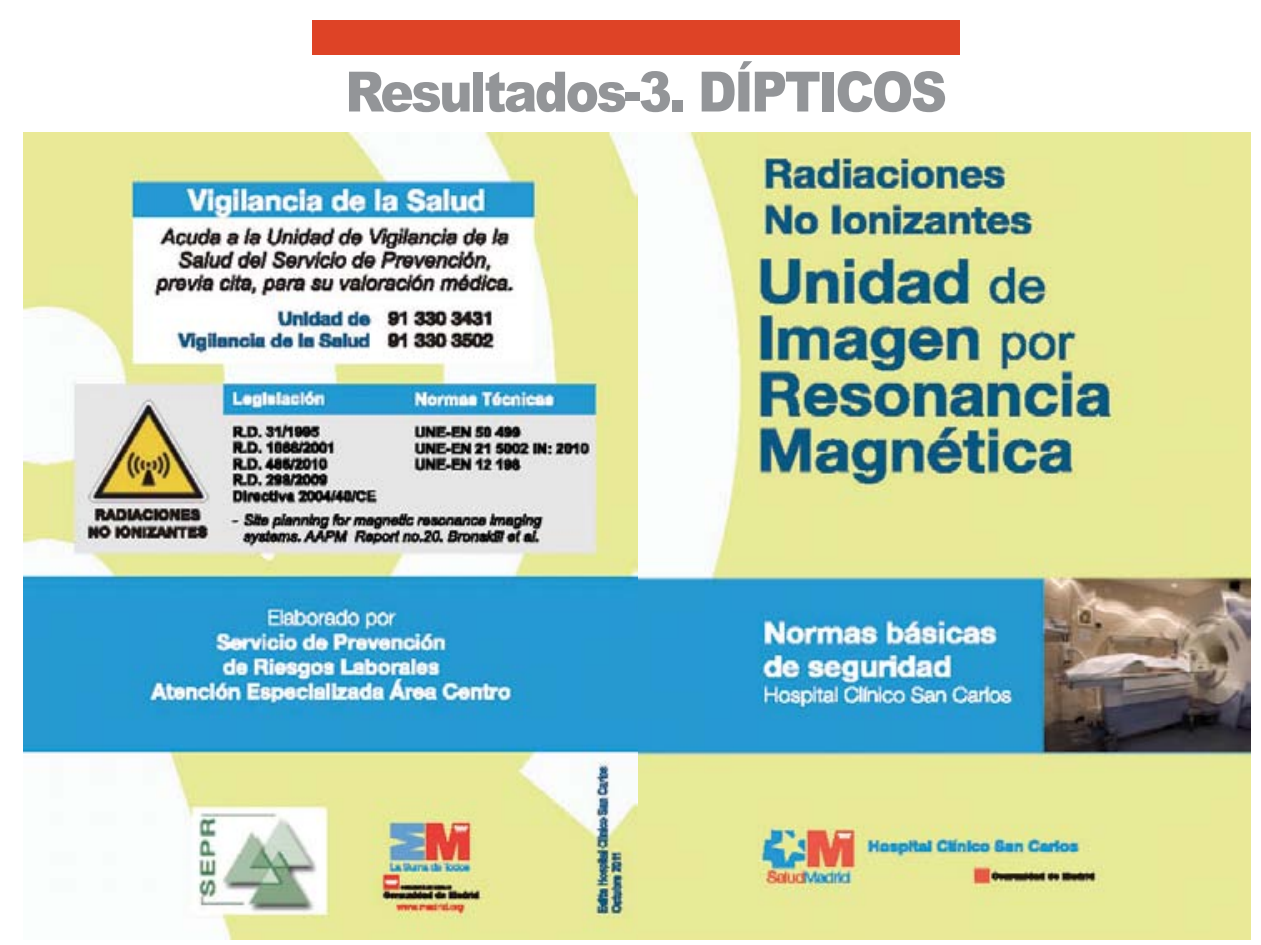




\section{Resultados-3. DÍPTICOS}

La Radiación No lonizante (RNI) se encuentra tanto en fuentes naturales (luz solar), como artificiales (luz eléctrica, radio, láser, infrarrojo, ultravioleta.......) y carece de energia para ionizar.

Desde el Servicio de Prevención y en colaboración con el Servicio de Física Médica se han elaborado unas normas básicas de seguridad en relación con I posible exposición ocupacional a RNI de los trabajadores de Atención

\section{Imagen por}

\section{Resonancia Magnética}

Es una prueba diagnóstica no invasiva que utiliza combinaciones de campo magnético estático y campos electromagnéticos de radiofrecuencias cuerpo de una persona.

Aplicaclones: Sinve para diagnosticar diferentes enfermedadeso estados patológicos mediante la obtención de imágenes anatómicas de alta definición sin necesidad de utilizar rayos $X$.

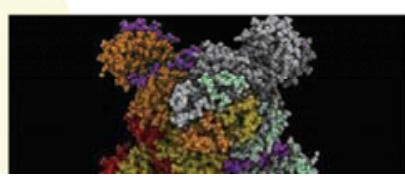

Medidas de control:

Trabajadores

- Mantener la puerta de la sala cerrada durante la

exploración.

- No permanecer de forma rutinaria en las proximidades del equipo, aunque se admite, por motivos de persona sanitario de forma esporádica y durante .

- EPI,S: El personal de la los trabajadores. que manipula los liquidos criogénicos, deberá esta debidamente entrenado y hacer uso de máscaras y guantes de protección

Los trabajadores especialmente sensibles: embarazadas, portadores de marcapasos u otros recibir valoración individualizada por el Servicio de Prevención.

Equipos

- Evitar la presencia de materiales ferromagnéticos en las proximidades del equipo: No introducir en la sala, material quirúrgico, relojes, ilaves u otros objetos ferro-magnéticos. Evitar e mantenimiento, extinción de incendios, etcr. Tomar precauciones especiales con mobiliario metálico (carritos o bandejas) y con las bombonas degases (oxigeno, $\mathrm{CO}_{\text {, }}$ )

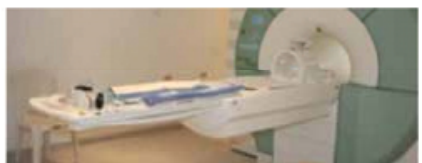

\section{Resultados-3. DÍPTICOS}

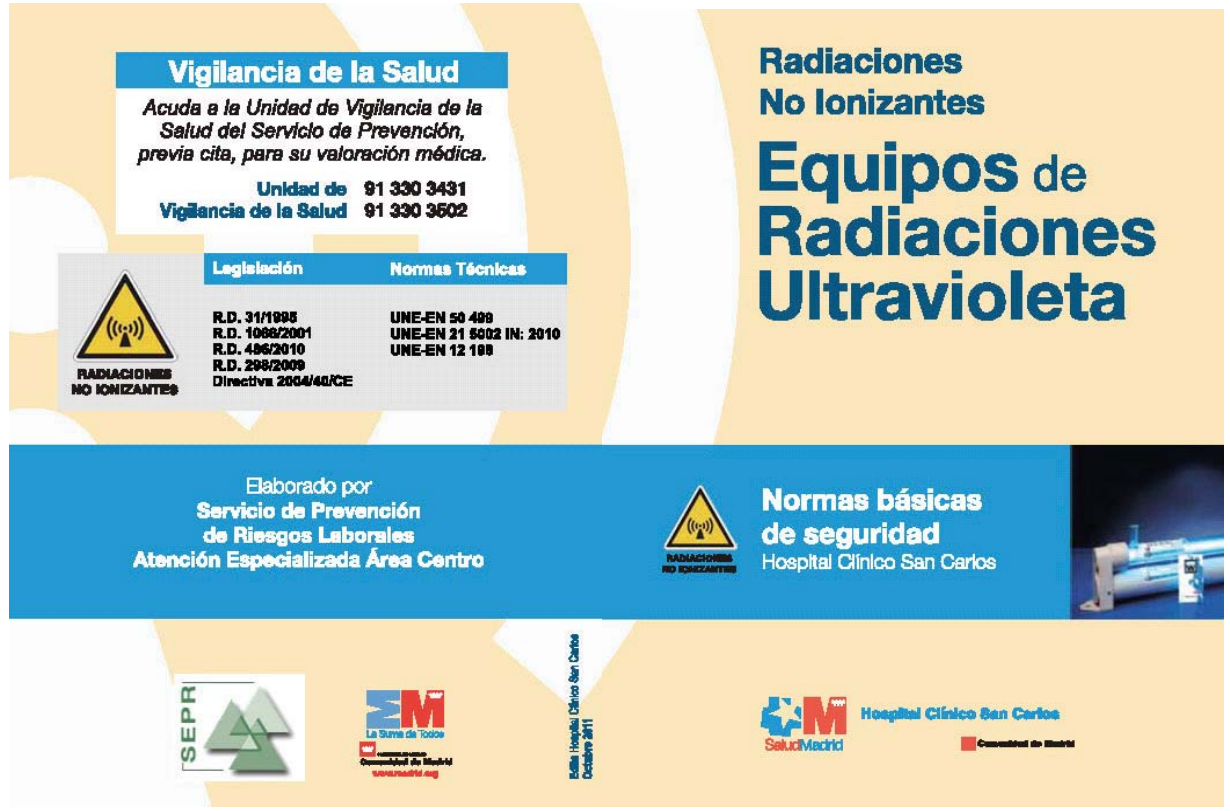




\section{Resultados-3. DÍPTICOS}

La Radiación No lonizante (RNI) se encuentra tanto en fuentes naturales (luz solar), como artificiales (luz eléctrica, radio, laser, infrarrojo, ultravioleta......) y carece de energla para ioniza. Desde el Servicio de Prevención y en colaboración con el Servicio de Fisica Médic se han elaborado unas normas básicas de. seguridad en relacion con la posible exposicion Atención Especialio los frea Codros

Radiaciones Ultravioleta (RUV)

Es una forma de radiación electromagnética con longitudes de onda mas cortas y fotones mas energetcosque los dela luz visible.

Lestos RUV solo se detecta por el resplandor de materiales que producen fluorescencia.

Aplicaciones

Son utilizadas en los campos de la Ciencia y la Medicina. Se aplican en tratamientos de algunas colencias cutaneas. Las RUV pueden uti zarse fotosensibilizadores.

Clasificación UVA, UVBYUVC.

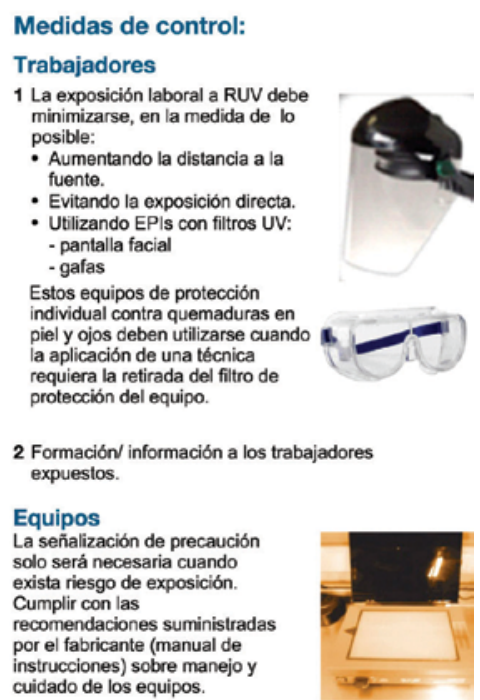

\section{Resultados-3. DÍPTICOS}

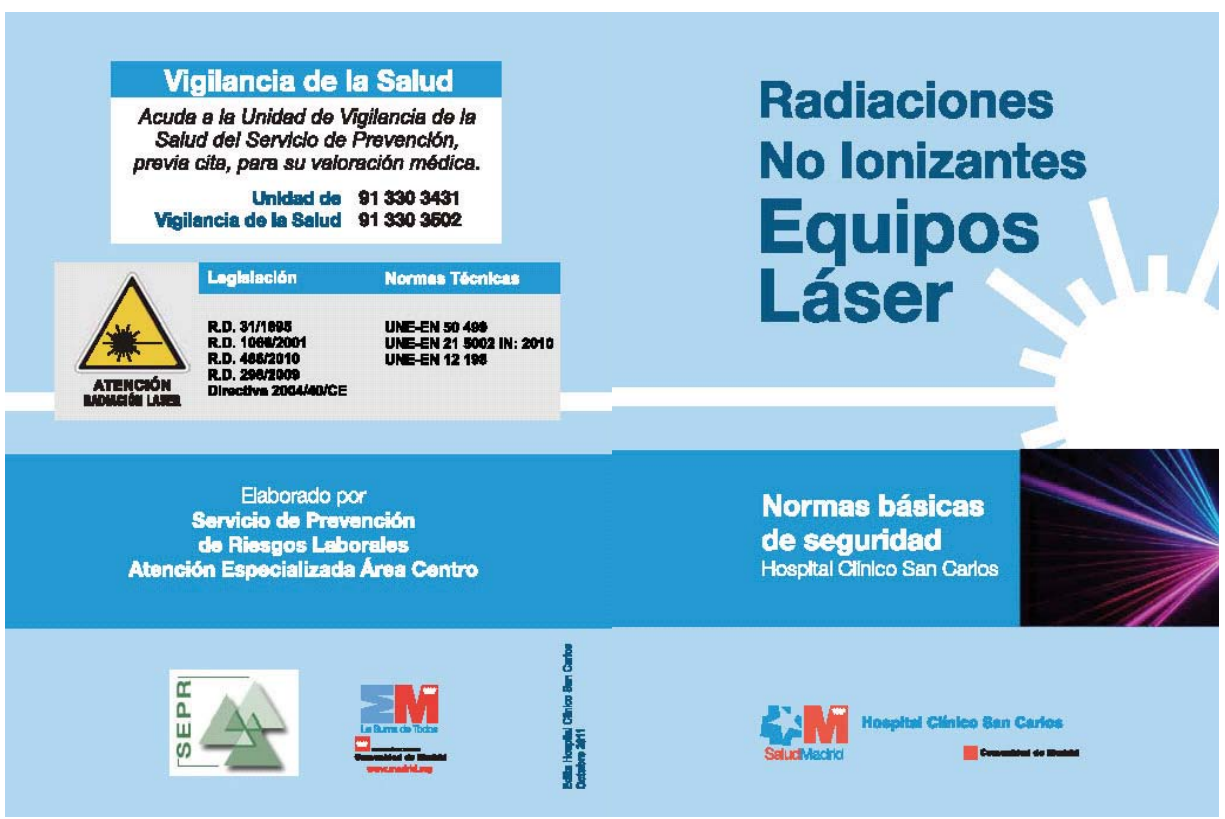




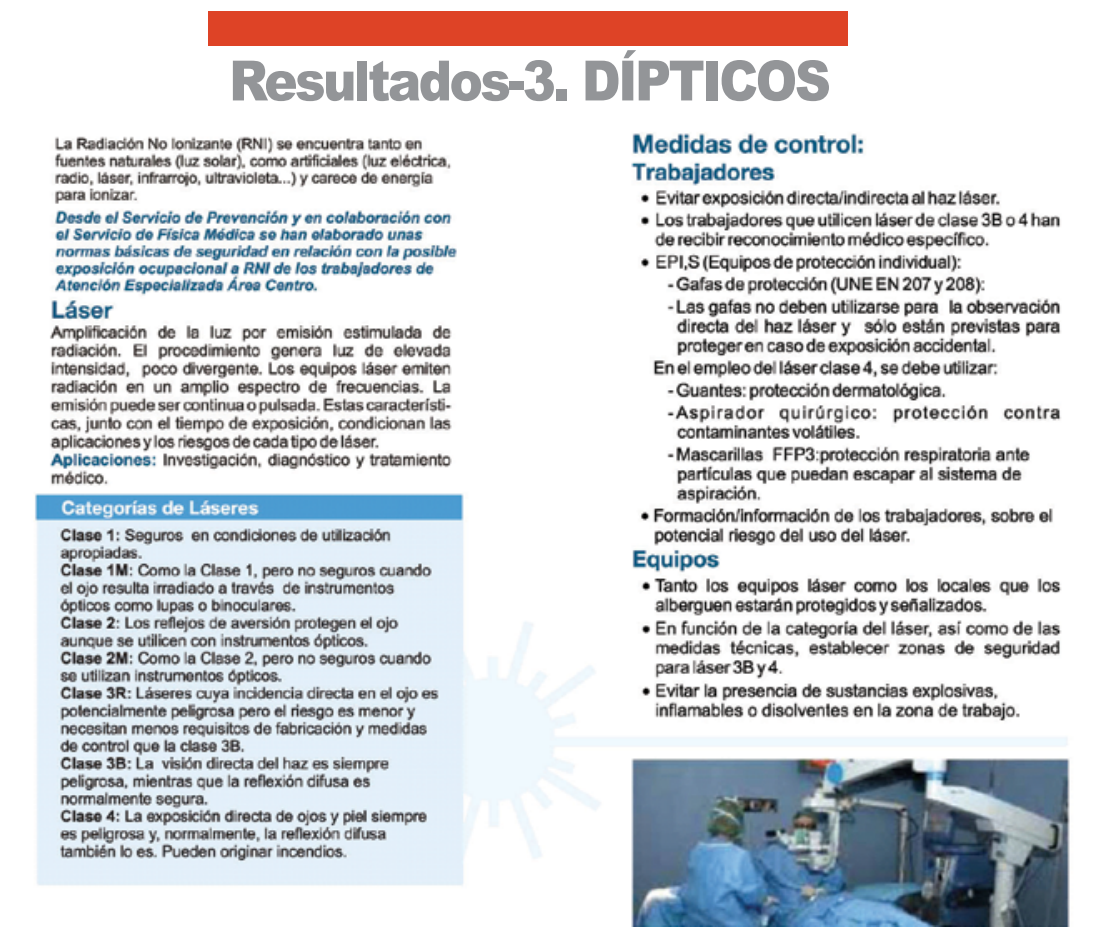

\section{Resultados-3. DIPTICOS}

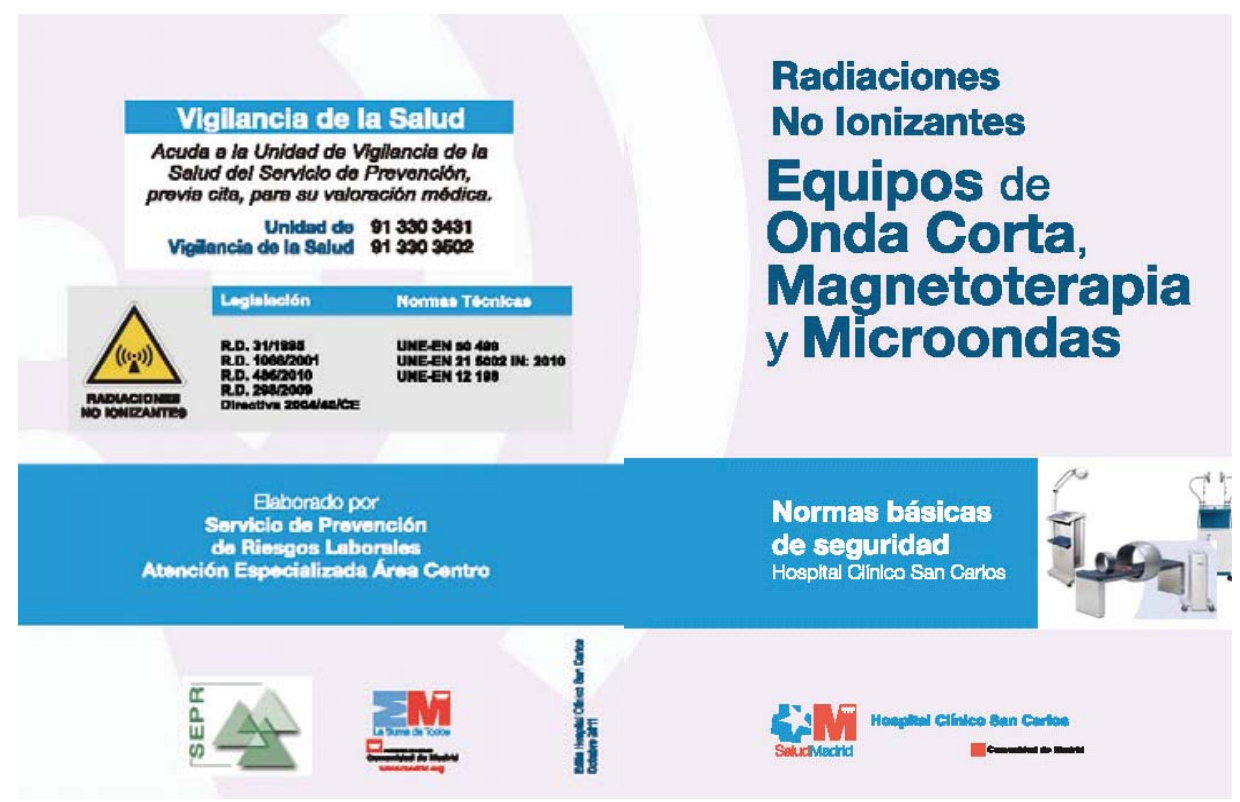




\section{Resultados-3. DíPTICOS}

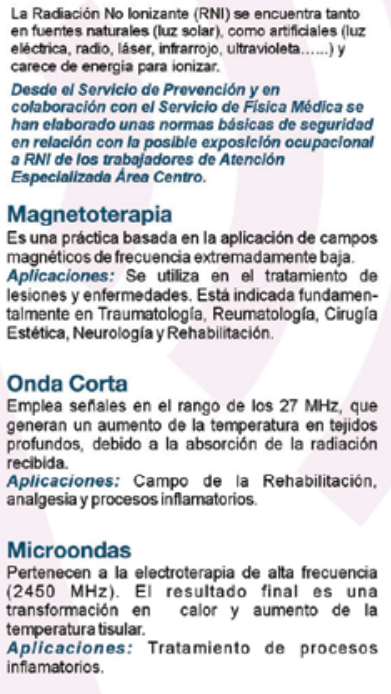

Medidas de control:

Trabajadores

- La exposición laboral, a onda corta, magnetoterapia

posible:

- Estableciendo una distancia de seguridad no inferior a un metro evitar portar objetos metálicos (relojes.

- Los trabajadores especialmente sensibles: implantes activos, 0 recibir valoración individualizada por el Servicio de Prevención

Equipos

- Mantener una distancia de seguridad no inferior a 2 metros entre equipos

pia no debe de ponerse en habitaciones húmedas salas de hidroterapia. - Evitar la exposición de los equipos a luz directa, solar - UV. Evitar la condensación de humedad sobre los salas de hidroterapia o de cabinas de rayos UVY Realización del mantenimiento preventivo de los equipos.

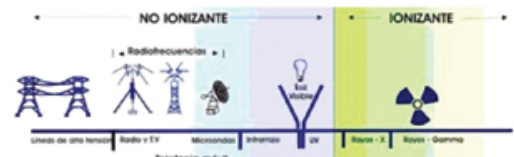

tकinem

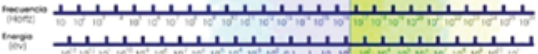

Aplicaciones: Tratamiento de procesos inflamatorios.

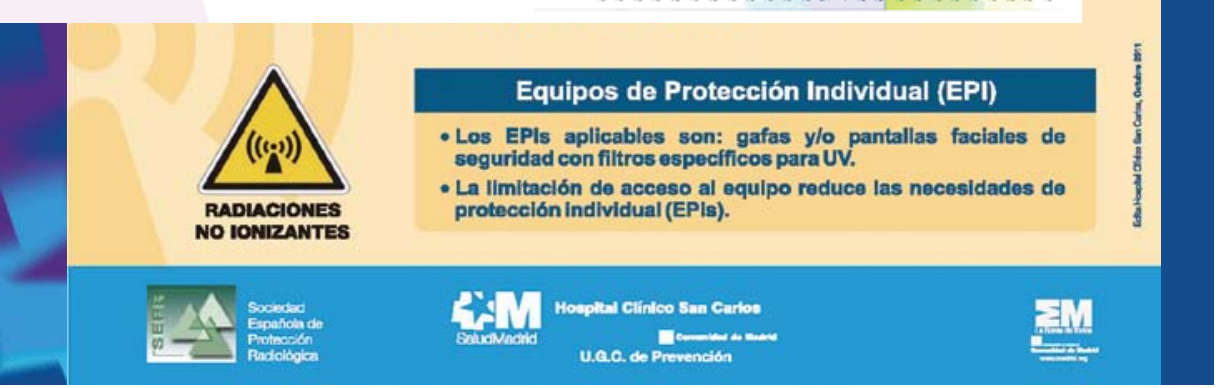




\section{Resultados-3. CARTELES}

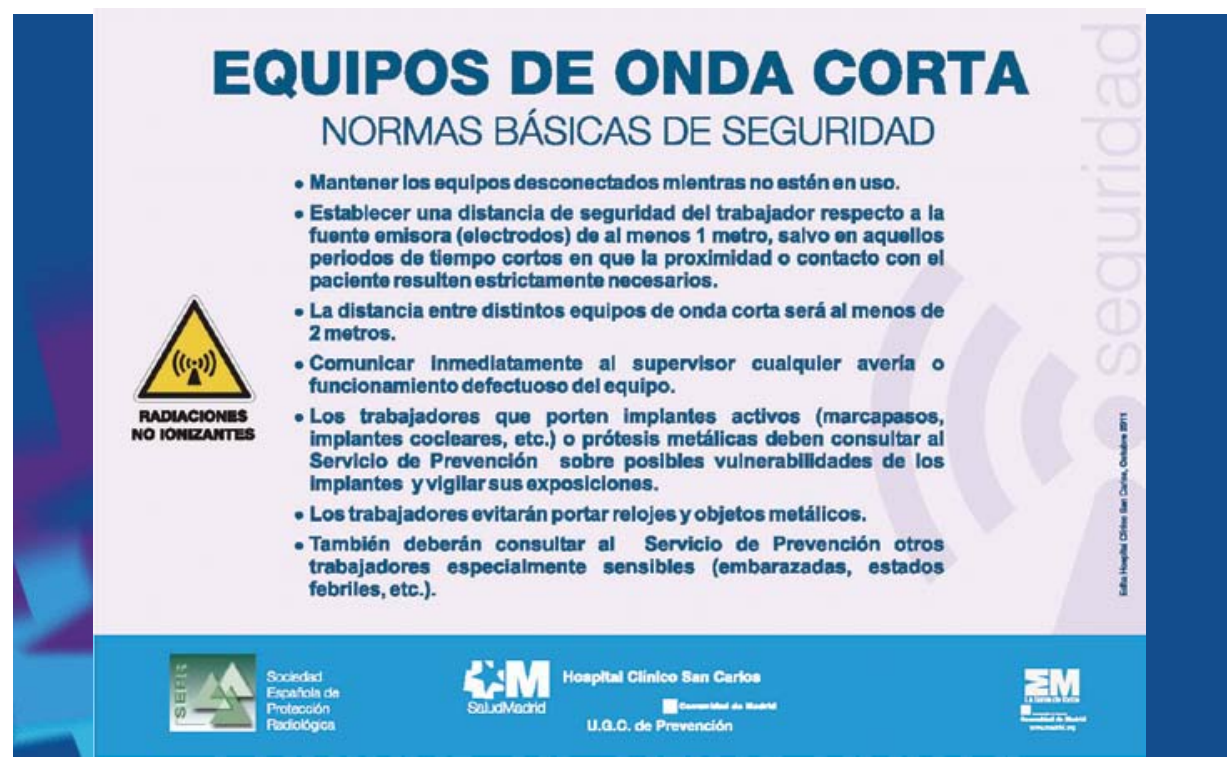

\section{Resultados-3. CARTELES}

\section{EQUIPOS MICROONDAS}

\section{NORMAS BÁSICAS DE SEGURIDAD}

- Mantener los equipos desconectados mlentras no estén en uso.

- Establecer una distancia de seguridad del trabajador respecto a la fuente emisora (electrodos) de al menos 1 metro, salvo en aquellos periodos de tlempo cortos en que la proximidad o contacto con el paclente resulten estrictamente necesarios.

- La distancia de seguridad entre distintos equipos de microondas será al menos de 2 metros.

- Comunicar inmediatamente al supervisor cualquier avería o

RADIACIONES funcionamiento defectuoso del equipo.
NO IONIZANTES - Los trabajadores que porten implantes activos (marcapasos, implantes cocleares, etc.) o prótesis metallicas deben consultar al Servicio de Prevención sobre posibles vulnerabilidades de los implantes y vigilar sus exposiciones.

- Los trabajadores evitarán portar relojes y objetos metálicos.

- También deberán consultar al Servicio de Prevención otros trabajadores especialmente sensibles (embarazadas, estados febriles, etc.). 


\section{Resultados-3. CARTELES}

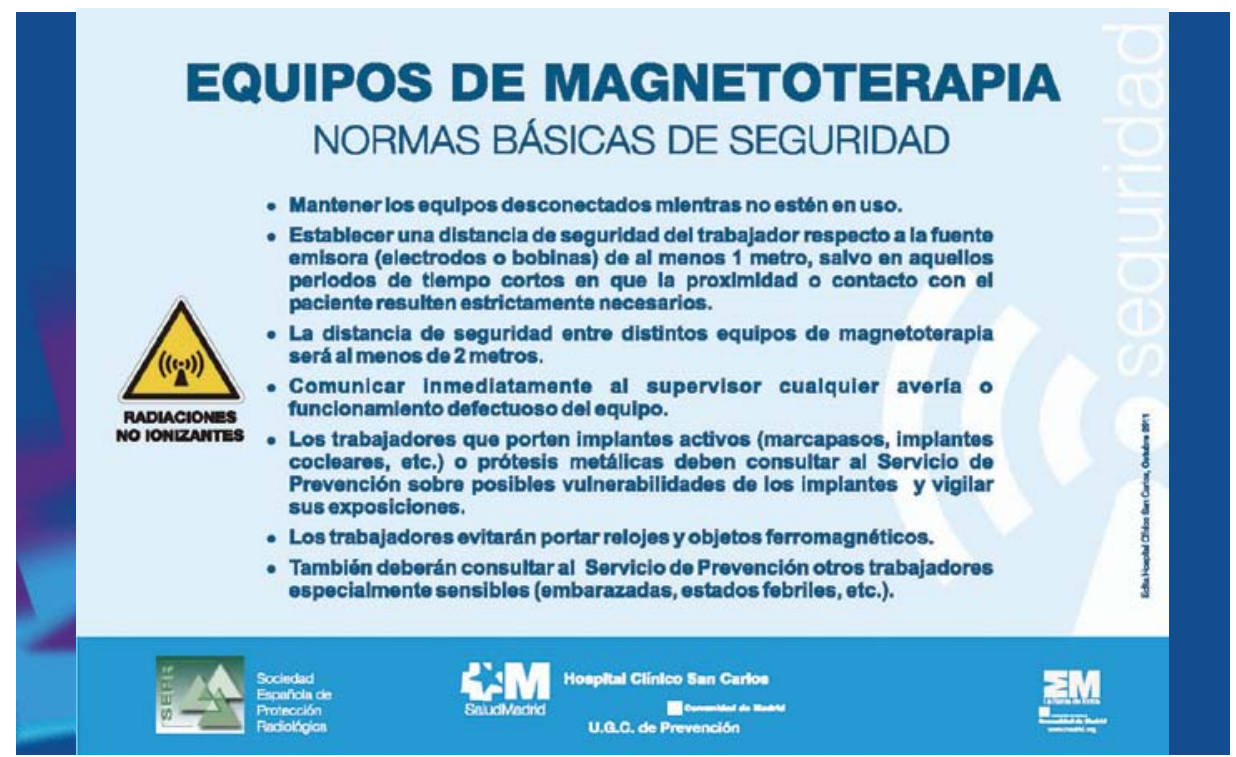

Resultados-3. CARTELES

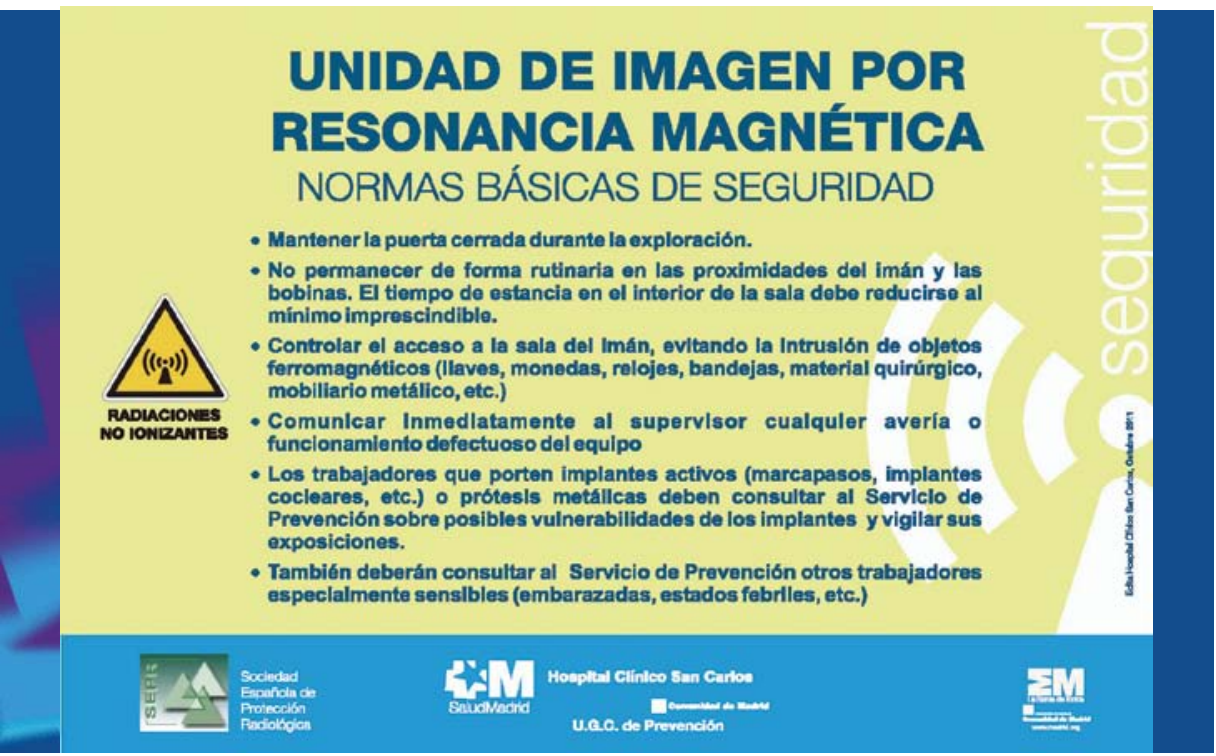




\section{Resultados-3. CARTELES}

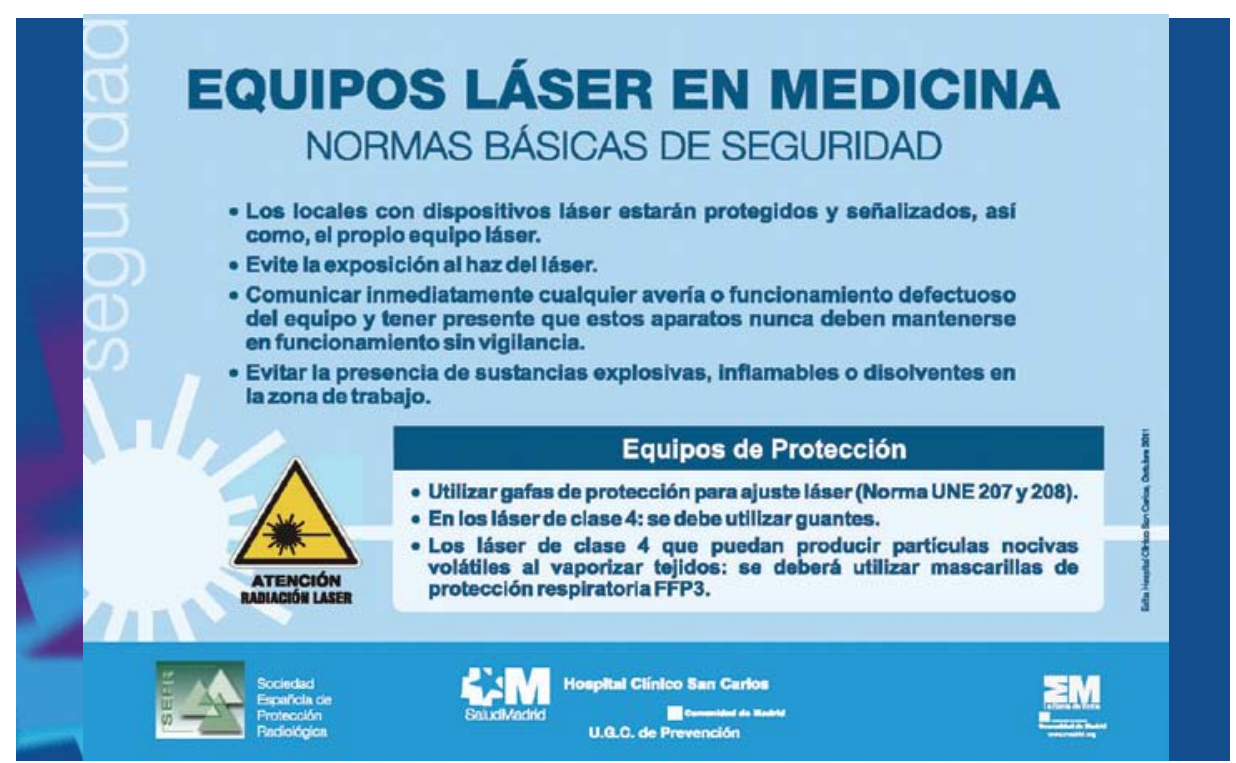

\section{CONCLUSIONES}

Hasta el momento no se ha identificado ningún mecanismo biológico que muestre una posible relación causal entre la exposición a CEM y el riesgo de padecer alguna enfermedad.

No está demostrado actualmente que la exposición a campos electromagnéticos ocasione efectos adversos para la salud, cumpliéndose los niveles de seguridad establecidos por ICNIRP.

Por otra parte, los trabajadores muestran un creciente interés, por las posibles consecuencias de la exposición humana a Radiaciones No Ionizantes.

En este contexto, nuestro Servicio de Prevención establece un Procedimiento para poner los medios que faciliten el cumplimiento de las recomendaciones de ICNIRP.

La elaboración del Procedimiento y su posterior implantación, nos permite:

- Asegurar el correcto manejo de los equipos en condiciones de seguridad.

- La adopción de medidas de protección individual y colectiva.

- Reforzar la herramienta de la formación/información de los trabajadores sobre Radiaciones No Ionizantes.

- Las medidas adoptadas con los equipos de trabajo (revisiones preventivas y correctivas), nos garantizan sus condiciones de seguridad.

- El Procedimiento fomenta la vigilancia y control de la salud de estos trabajadores.

- Por ultimo, este Procedimiento contribuye a la integración de la prevención, ya que los trabajadores lo perciben como un compromiso de la organización con su salud y como una oportunidad para aportar su conocimiento y experiencia en la mejora de su calidad laboral. Todo ello incrementa su compromiso con propia seguridad. 


\section{AGRADECIMIENTOS}

A D. Alejandro Ubeda-Maeso, Investigador Jefe de Sección, Servicio de InvestigaciónBEM. Hospital Ramón y Cajal-IRYCIS.

A D. Jesús García Aranda, Diseño Gráfico, Hospital Clínico San Carlos.

\section{REFERENCIAS BIBLIOGRÁFICAS}

\section{Legislación}

1. LEY 31/1995, de Prevención de Riesgos Laborales.

2. R.D. 1066/2001 por el que se aprueba el Reglamento que establece condiciones de protección del dominio público radioeléctrico, restricciones a las emisiones radioeléctricas y medidas de protección sanitaria frente a emisiones radioeléctricas.

3. REAL DECRETO 298/2009, de 6 de marzo, por el que se modifica el Real Decreto 39/1997, de 17 de enero, por el que se aprueba el Reglamento de los Servicios de Prevención, en relación con la aplicación de medidas para promover la mejora de la seguridad y de la salud en el trabajo de la trabajadora embarazada, que haya dado a luz o en período.

4. DIRECTIVA 2004/40/CE del Parlamento Europeo y del Consejo, de 29 de abril de 2004, sobre las disposiciones mínimas de seguridad y de salud relativas a la exposición de los trabajadores a los riesgos derivados de los agentes físicos (campos electromagnéticos) (decimoctava Directiva específica con arreglo al apartado 1 del artículo 16 de la Directiva 89/391/CEE).

5. R.D 486/2010 Protección de la Salud y la Seguridad de los trabajadores contra los riesgos relacionados con las radiaciones ópticas artificiales.

\section{Normas técnicas}

6. UNE-EN 50499 Procedimiento para la evaluación de la exposición de los trabajadores a los campos electromagnéticos.

7. UNE-EN 21502 IN: 2010 guía para la evaluación de la exposición de los trabajadores acampos electromagnéticos en relación con la Directiva 2004-40-CE.

8. UNE-EN 12198 evaluación y reducción de los riesgos debido a las radiaciones emitidas por las máquinas. Reducción de radiaciones mediante atenuamiento o apantallamiento.

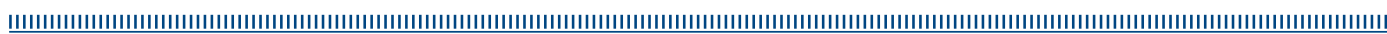

